\title{
Performance Analysis of IEEE 802.15.4/Zigbee Networks Under Route Information Based AODV Routing Algorithm
}

\author{
Rohit Ranjan ${ }^{1}$ Harsukhpreet Singh ${ }^{2}$ and Anurag Sharma ${ }^{3}$ \\ ${ }^{1,2,3}$ Department of electronics and communication \\ CTITR \\ jalandhar, India \\ ${ }^{1}$ Rohit.ranjaan85@gmail.com \\ 2harsukhpreet@gmail.com \\ Er.anurags@gmail.com
}

\begin{abstract}
In the field of wireless communication, zigbee is a latest technology in wireless network based on IEEE 802.15.4 standard. On IEEE 802.15.4 standard based zigbee network uses low power sensor nodes to ensure reliable, process, collect, and store the data. The sensor nodes have low cost, smaller in memory size and used in lower coverage area first two layers based on IEEE 802.15.4 standard are PHY and MAC layer. Routing protocols are divided in two categories that are reactive protocol and proactive protocol. $A O D V$ routing protocol comes under reactive protocol. It creates route between source and destination when needed. When source node has data to transmit to the destination node then it will create RREQ (route request message) and send to all neighbors in the network, when the destination node found or neighbor node found the route to destination then they will generate a RREP (route reply message) and forward back to source node. After the creation of route between source node and destination node they can exchange the data.
\end{abstract}

Keywords: IEEE 802.15.4, zigbee, AODV Routing, OPNET 14.5

\section{Introduction}

In the field of wireless communication, it uses low power sensor nodes to process, collect, and store the data. The sensor nodes have low cost, smaller in memory size and used in lower coverage area [8]. In wireless sensor network, the nodes can be more than thousands sensor nodes in a network. More number of sensors can sense larger area. zigbee is a latest technology in wireless network based on IEEE 802.15.4 standard. On IEEE 802.15.4 standard based zigbee network uses low power sensor nodes to ensure reliable, process, collect, and store the data [1]. The sensor nodes have low cost, smaller in memory size and used in lower coverage area first two layers based on IEEE 802.15.4 standard are PHY and MAC layer. The work done by both the layer are physical layer is responsible for detection of energy, calculation of signal quality, and reception and transmission of data. MAC layer is responsible establish route and route management between source node and destination node. It also handles the collision in the network. IEEE 802.15.4 based zigbee network uses $2.4 \mathrm{GHz}$ frequency band for communication. The frequency band $2.4 \mathrm{GHz}$ is license free band to use. Figure1 shows the protocol stack of IEEE 802.15.4 zigbee network [5].

\subsection{IEEE 802.15.4 ZIGBEE AD-HOC Routing}

In zigbee protocol stack, first two layers based on IEEE 802.15.4 standard are PHY and MAC layer. The work done by both the layer are physical layer is responsible for 
detection of energy, calculation of signal quality, and reception and transmission of data. MAC layer is responsible establish route and route management between source node and destination node [10]. It also handles the collision in the network. IEEE 802.15.4 based zigbee network uses $2.4 \mathrm{GHz}$ frequency band for communication. The frequency band 2.4 $\mathrm{GHz}$ is licence free band to use. Figure1 shows the protocol stack of IEEE 802.15.4 zigbee network $[2,3]$.

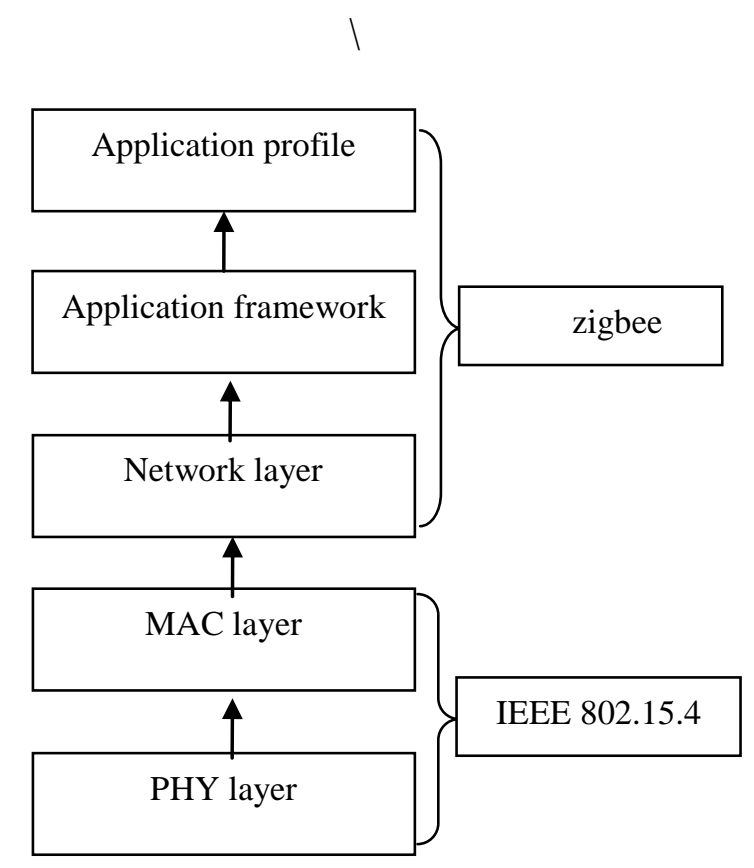

Figure 1. Protocol Stack [5]

Coordinator - it is the backbone of the network which is used to control the whole network. It has capability to save the information about the devices within the network which is used. Coordinator of network cannot sleep; it will be always active.

Router - it is used to provide route information between sources to destination in the network. Router gives the shortest path between source and destination.

End device - it is used to transmission and reception of data whenever source user and the destination user have data to transmit. End devices can only transmit or receive, cannot provide route information to the network [4].

Star topology- in the star topology, end devices cannot send data directly to the other devices, data of each device can go through coordinator to the 


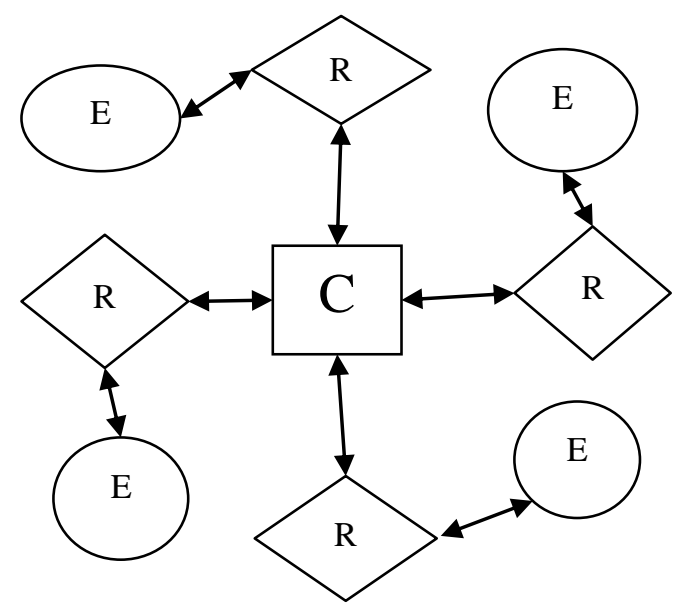

Figure 1. Star Network [5]

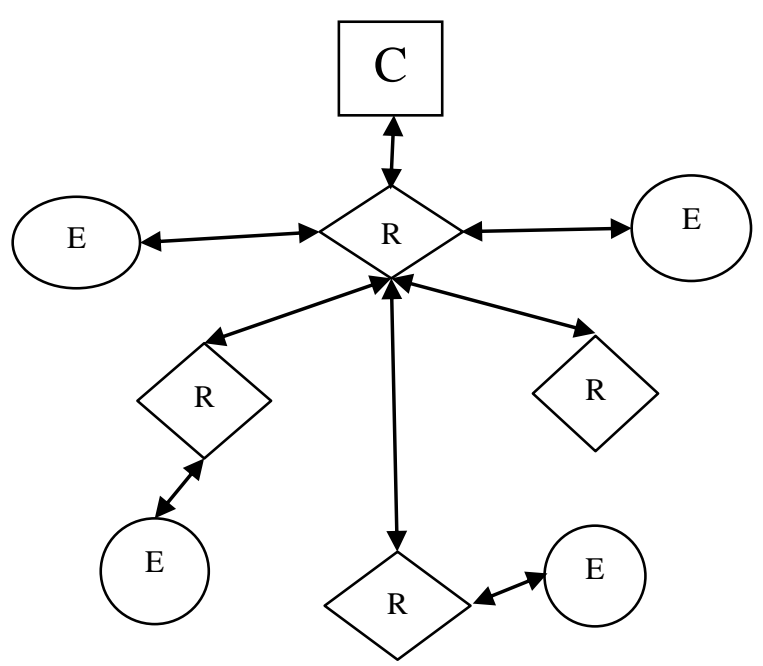

Figure 2. Tree Network [5]

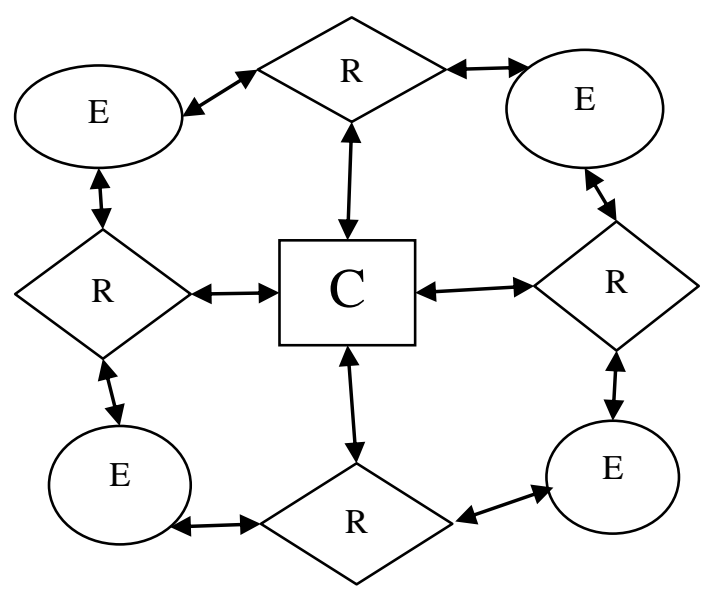

Figure 3. Mesh Network [5] 
other devices. If the coordinator broke down, then network will shut down and end devices can not transmit data.

Tree topology - it is the combination of bus topology and star topology. In which one node is called parent node and other are called child node. In tree topology, each node has point to point link to all node around to it. In the tree network, if one link is break than other link will be active.

Mesh topology - in mess topology of zigbee network, each node is connected to directly to other node through radio link so that if one link is break then data is transferred through other route. Due to this reason mess network of zigbee network has less delay [9].

Routing protocols are divided in two categories that is reactive protocol and proactive protocol. AODV routing protocol comes under reactive protocol. It create route when needed. When source node has data to transmit to the destination node then it will create RREQ (route request message) and send to all neighbors in the network, when the destination node found or neighbor node found the route to destination then they will generate a RREP (route reply message) and forward back to source node. After the creation of route between source node and destination node they can exchange the data [7, $17,14]$.

\section{Literature Survey}

Rajagopalan R. et al. [2013] discussed about the routing protocol which is AODV and DYMO. They concluded that AODV work better in zigbee network in terms of end to end delay through put and number of hops also said that performance of network can vary after increasing or decreasing the number of nodes. Kalaivani K. et al. [2015] discussed the method to integrate the zigbee network with $4 \mathrm{G}$ technology for fast data transmission and to provide better security to the network. Performance is compared in terms of data sent, jitter, delay, end to end delay, and packet delay. Stevanovic D. et al. [2008] compared the two topologies of zigbee network on the basis of random sink mobility and predictable sink mobility in the network and they concluded that random sink movement is better than predefined sink movement in zigbee network. Hammoodi I. et al. [2009] investigated the capability of OPNET modeller in zigbee. They suggested that OPNET modeller is suitable for zigbee but they also mentioned that modeller work well with MAC layer but in PHY layer modeller needs some improvement, they encountered that there is energy consuming problem. Nourildean S. et al. [2012] described and compared the performance of one coordinator and multiple coordinators in the zigbee network. They concluded that tree routing is better than mesh routing in zigbee network with multiple coordinators. Kaur A. et al. [2014] described the hybrid topologies in zigbee by using three topologies of zigbee on the basis of delay, throughput, end to end delay etc. and said that hybrid topologies is much better than simple topologies of zigbee. Hussein A. et al. [2015] described the location effect of coordinator in the zigbee network. They designed two networks on different location of coordinator and concluded that central location of coordinator in the network is more suitable than other. Kaur S. et al. [2016] compared and analyzed the performance of two routing topologies that was tree and mesh routing. They concluded that tree routing is better than mesh in terms of throughput but mesh routing has less delay than tree routing.

\section{Result and Discussion}

I compare the performance of zigbee mesh routing and AODV routing in mesh topology of zigbee network. Comparison performs on the basis of delay, throughput, and packet delivery ratio using OPNET modeler 14.5. in the result, AR-Mesh (AODV routing in mesh topology) gives better result than mesh topology on the basis of all parameter such as delay, throughput, and packet delivery ratio. AODV create route when needed. When source node has data to transmit to the destination node then it will create RREQ 
(route request message) and send to all neighbors in the network, when the destination node found or neighbor node found the route to destination then they will generate a RREP (route reply message) and forward back to source node. After the creation of route between source node and destination node they can exchange the data. Figure 4 shows the comparative graph of zigbee mesh routing and AR-Mesh routing on the basis of throughput. Throughput of AR-Mesh routing in zigbee network is maximum because AODV create route when needed, when source node has data to transmit to the destination node then it will create RREQ and send to all neighbours in the network then load of networks are divided among the entire router and the coordinator and due to this reason collision reduced in the network. data traffic received at the receiver side is the traffic of data on the whole network. The result is given in bits/sec. figure 5 shows the comparative graph of zigbee mesh routing and AR-Mesh routing on the basis of Delay, delay is combination of all delays in the network generated by route maintenance process, queuing, retransmission due to failure link and propagation of data from source to destination. Delay is maximum in AR-mesh routing of zigbee network because data is sent one node to another node from source to destination in the network and minimum in the mesh network. Delay in the network, generated by route maintenance process, queuing, retransmission due to failure link and propagation of data from source to destination. Figure 6 shows the comparative graph of zigbee mesh routing and AR-Mesh routing on the basis of PDR. packet delivery ratio is the ratio of data traffic received divided by data traffic sent. In which, data traffic sent is the traffic of data sent by sender side on the network and data traffic received is traffic received at the receiver side, PDR of AR-Mesh routing in zigbee network is maximum because AODV create route when needed, When source node has data to transmit to the destination node then it will create RREQ and send to all neighbours in the network then load of networks are divided among the entire router and the coordinator and due to this reason collision reduced in the network. data traffic received at the receiver side are maximum. 


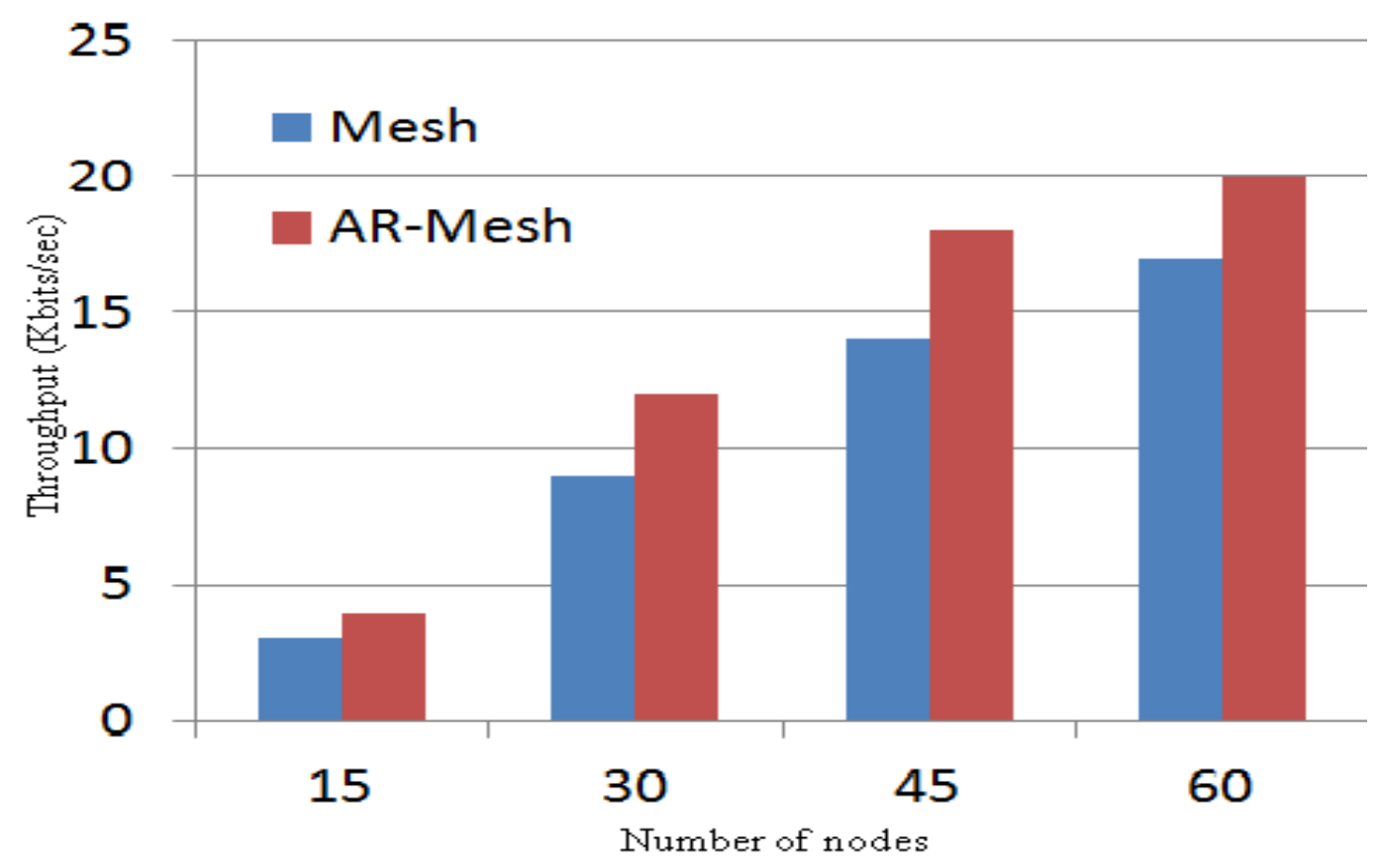

Figure 4. Throughput

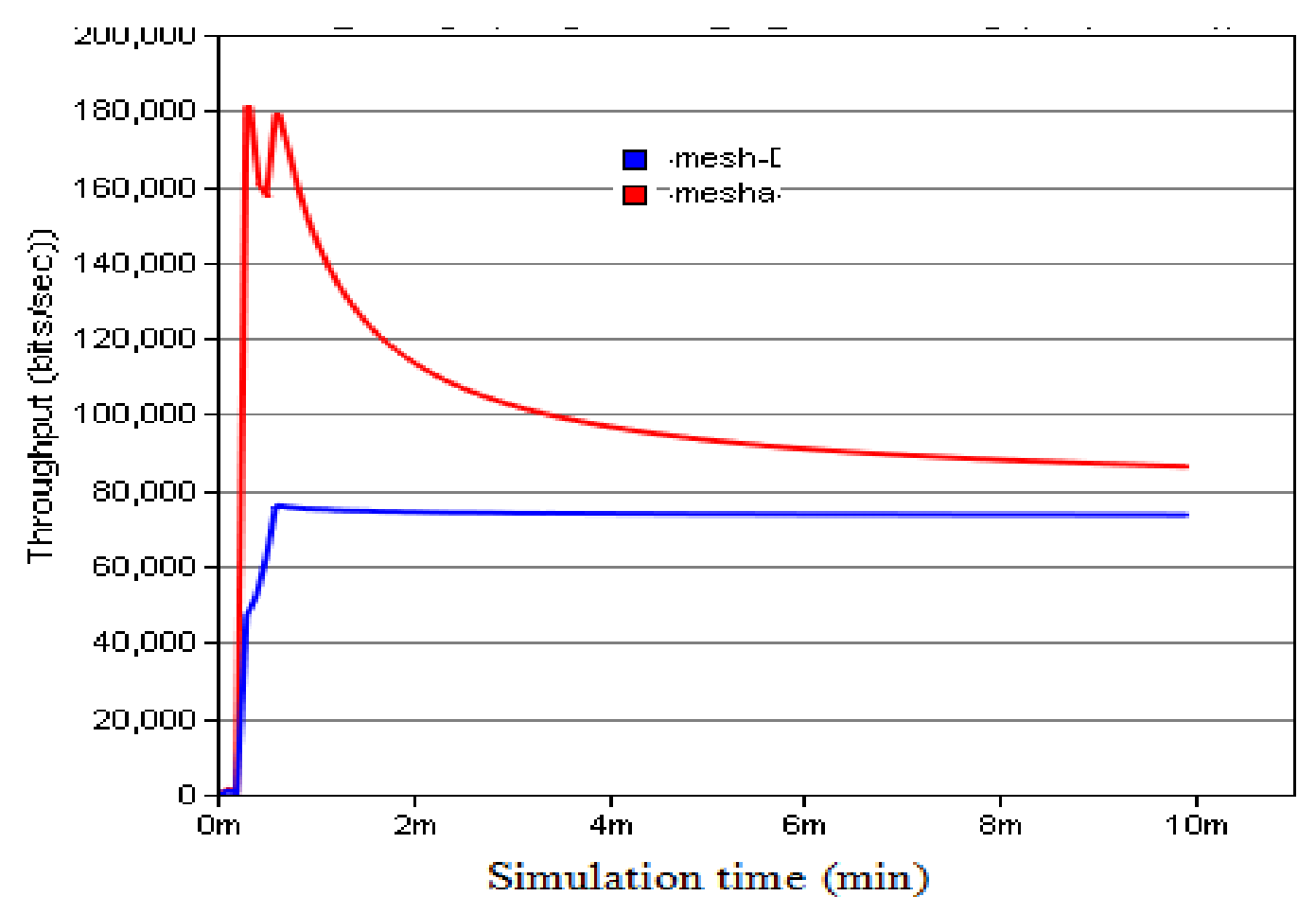

Figure 5. Throughput 


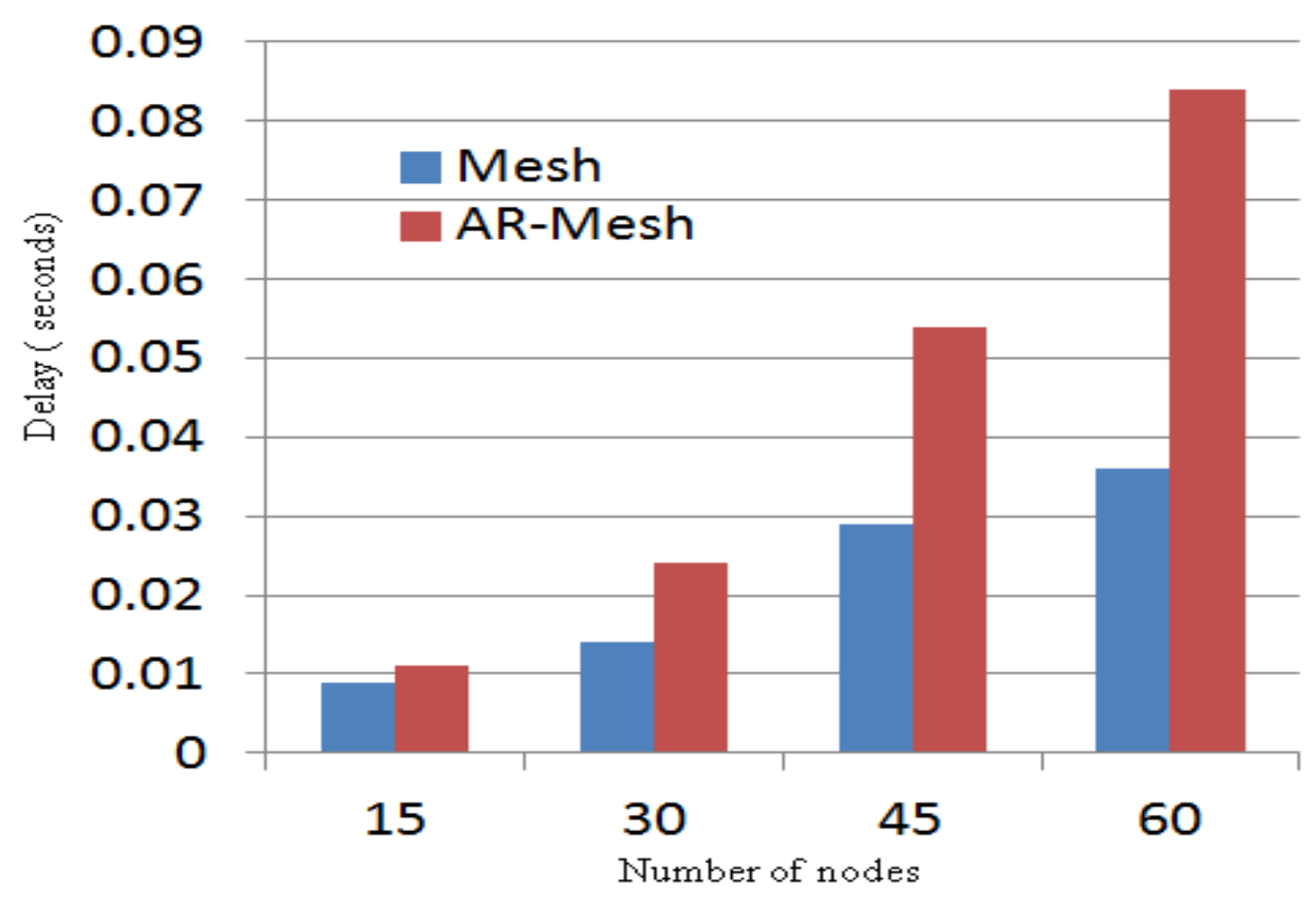

Figure 6. Delay

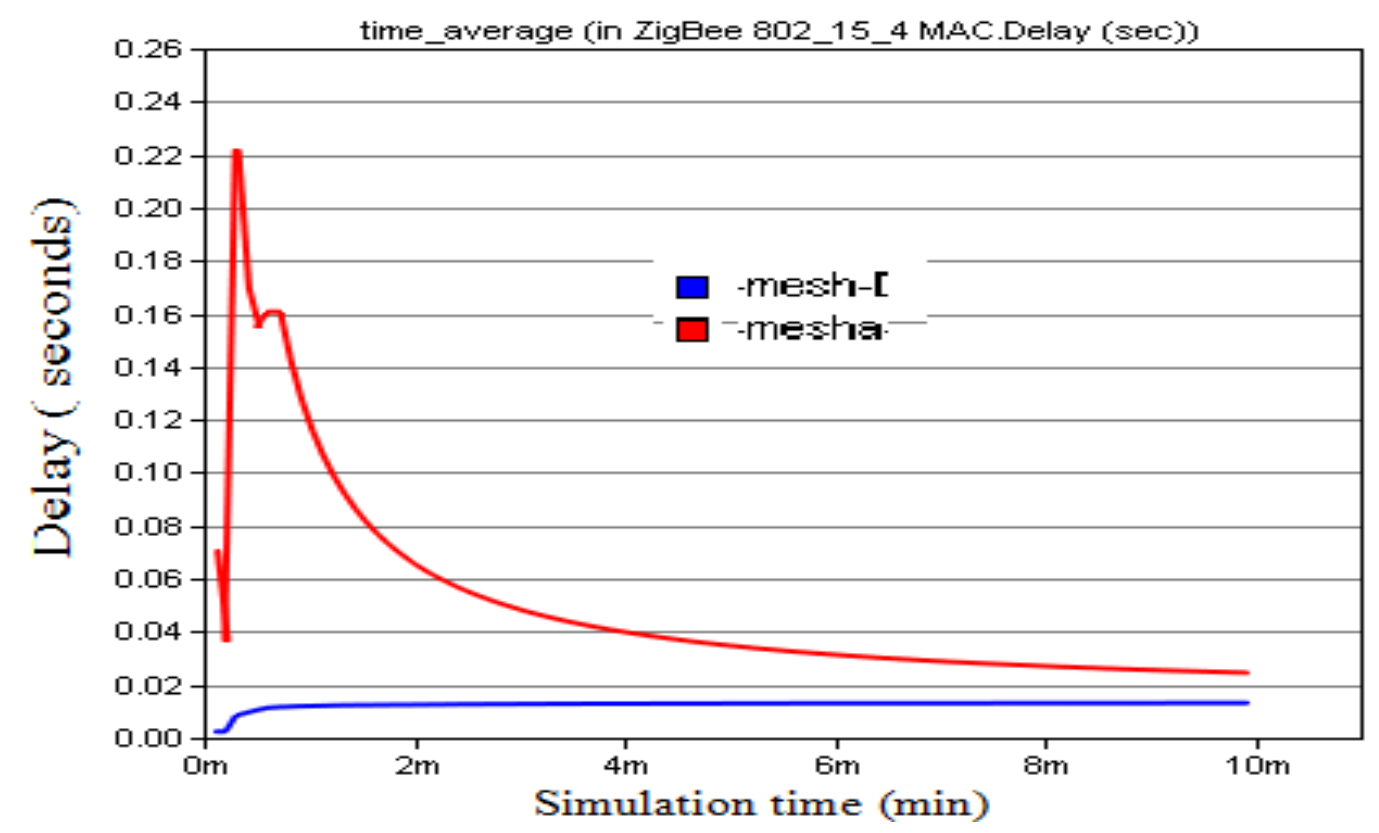

Figure 7. Delay 


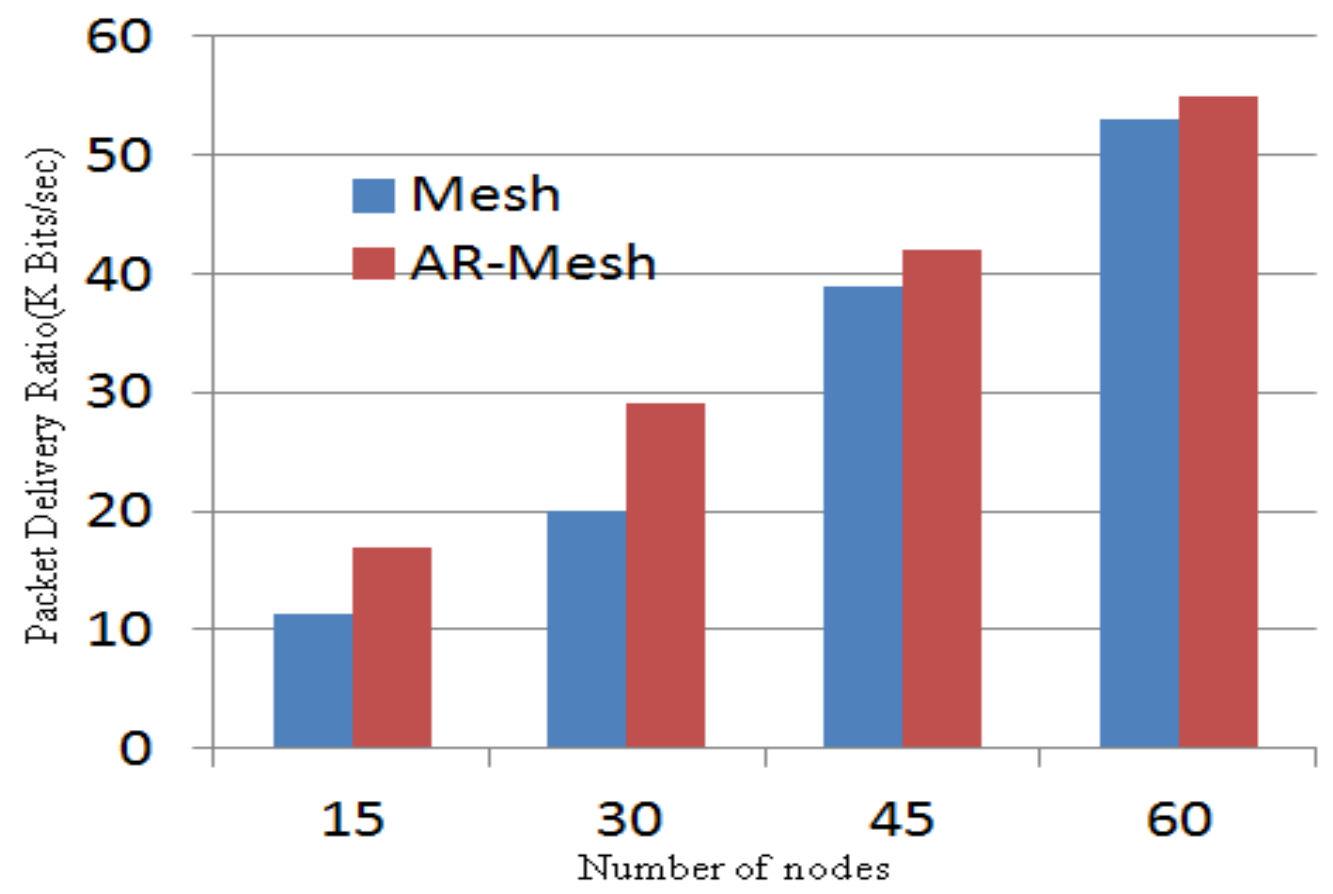

Figure 8. Packet Delivery Ratio

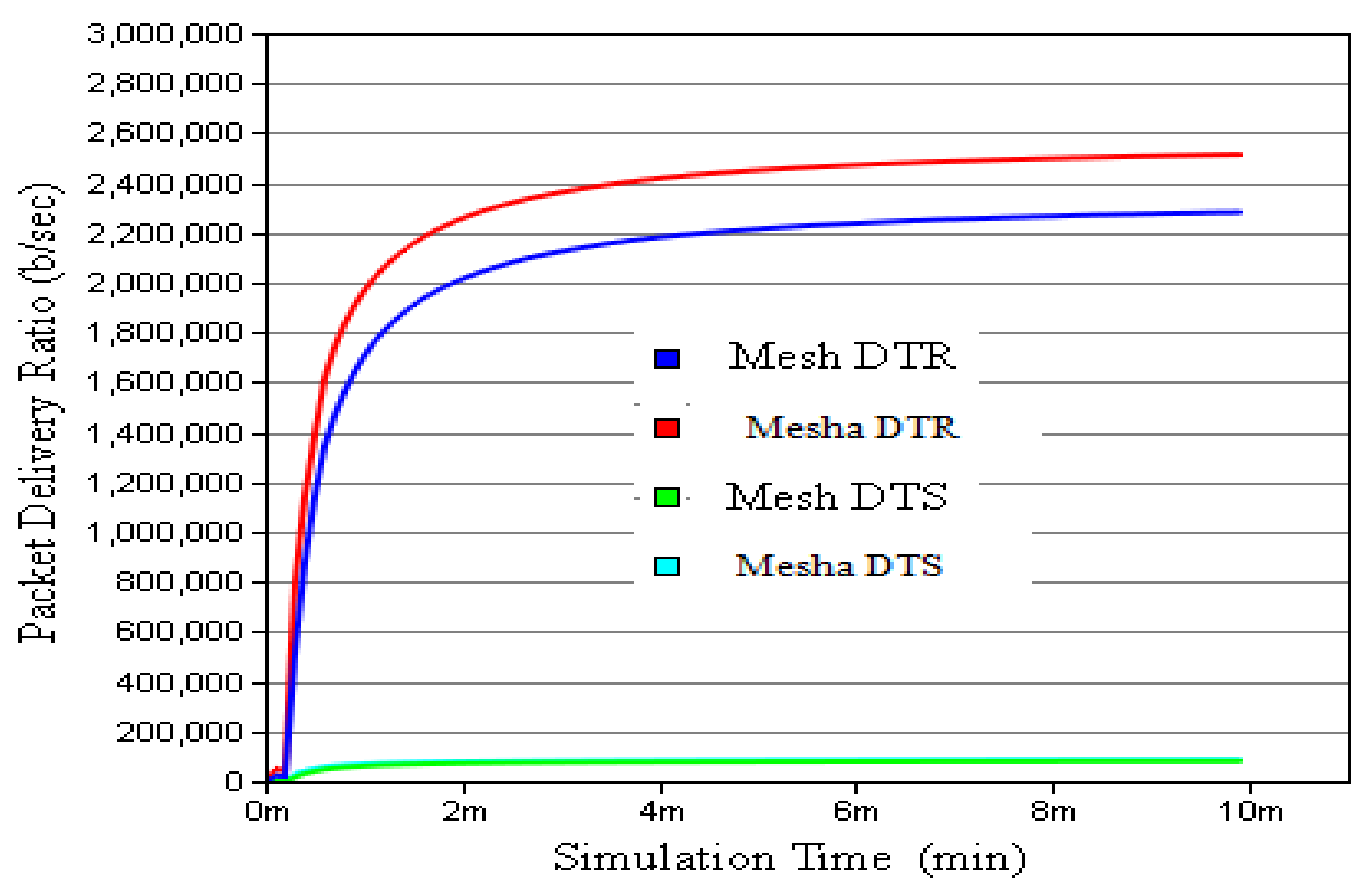

Figure 9. Packet Delivery Ratio 


\section{Table of Results}

\begin{tabular}{|c|c|c|c|c|c|c|c|}
\hline \multirow{2}{*}{ Number of nodes } & \multicolumn{2}{|c|}{ Delay (sec) } & \multicolumn{2}{|c|}{ Throughput (kb) } & \multicolumn{2}{c|}{ PDR (kb) } \\
\cline { 2 - 7 } & Mesh & $\begin{array}{c}\text { AODV } \\
\text { Mesh }\end{array}$ & Mesh & $\begin{array}{c}\text { AODV } \\
\text { Mesh }\end{array}$ & Mesh & $\begin{array}{c}\text { AODV } \\
\text { Mesh }\end{array}$ \\
\hline 15 Nodes & .0092 & .011 & 3 & 4 & 11.25 & 17 \\
\hline 30 Nodes & 0.012 & 0.024 & 9 & 13 & 20 & 29 \\
\hline 45 Nodes & 0.019 & 0.054 & 14 & 18 & 39 & 42 \\
\hline 60 Nodes & 0.025 & 0.084 & 17 & 20 & 53 & 55 \\
\hline
\end{tabular}

\section{Conclusion}

In this paper, we compare the performance of zigbee mesh routing and AODV routing in mesh topology of zigbee network. Comparison performs on the basis of delay, throughput, and packet delivery ratio using OPNET modeler 14.5. in the result, AR-Mesh (AODV routing in mesh topology) gives better result than mesh topology on the basis of all parameter such as delay, throughput, and packet delivery ratio. In the result, , ARMesh(AODV routing in mesh topology) has maximum delay, throughput, packet delivery ratio as compare to mesh topology. But for delay, mesh routing is more suitable than other topologies. zigbee is a latest technology in wireless network based on IEEE 802.15.4 standard. On IEEE 802.15.4 standard based zigbee network uses low power sensor nodes to ensure reliable, process, collect, and store the data [1]. The sensor nodes have low cost, smaller in memory size and used in lower coverage areafirst two layers based on IEEE 802.15.4 standard are PHY and MAC layer and AODV create route when needed. When source node has data to transmit to the destination node then it will create RREQ (route request message) and send to all neighbors in the network, when the destination node found or neighbor node found the route to destination then they will generate a RREP (route reply message) and forward back to source node. After the creation of route between source node and destination node they can exchange the data. 


\section{References}

[1] A. Bhatia and P. Kaushik, "A Cluster Based Minimum Battery Cost AODV Routing Using Multipath Route for ZigBee", international conference on network, proceeding of IEEE, (2008).

[2] D. Stevanovic and N. Vlajic, "Performance of IEEE 802.15.4 in Wireless Sensor Networks with a Mobile Sink Implementing Various Mobility Strategies", Conference on Local Computer Networks (LCN), proceeding of IEEE, (2008), pp. 680-688.

[3] W. Chunming and Z. Xuemei, "Routing Algorithm Simulation of The Patient Monitoring System Based on ZigBee", International Conference on Networking and Digital Society, proceeding of IEEE, (2010), pp. 26-29.

[4] M. Kasraoui, A. Cabani and J. Mouzna, "Improvement of Zigbee Routing Protocol" 2012 IEEE International Conference on Green Computing and Communications, Conference on Internet of Things, and Conference on Cyber, Physical and Social Computing, proceeding of IEEE, (2012), pp. 788-793.

[5] R. Rajagopalan and A. Dahlstrom, "Performance Analysis of Routing Protocols in Zigbee Non- Beacon Enabled WSNs", the internet of things: RFID, WSN and beyond, proceeding of IEEE, (2013).

[6] J. Singh and R. Mahajan, "Performance Analysis of AODV And OLSR Using OPNET" International Journal of Computer Trends and Technology (IJCTT), vol. 5, no. 3, (2013).

[7] S. Kaur, S. Singh and G. Singh, "Examine the Performance of different Topologies using Opnet 14.5 in ZigBee Sensor Network", International Journal of Computer Applications (0975 - 8887), vol. 108, no. 7, (2014).

[8] A. Malek, C. Li and L. Li, "Improving ZigBee AODV Mesh Routing Algorithm Topology and Simulation Analysis", TELKOMNIKA Indonesian Journal of Electrical Engineering, vol. 12, no. 2, (2014), pp. 1528- 1535.

[9] A. Hussein and G. Samara, "Coordinator Location Effects in AODV Routing Protocol in ZigBee Mesh Network", International Journal of Computer Applications (0975 - 8887), vol. 127, no. 8, (2015).

[10] K. Kalaivani, "Zigbee Sensor Network Integrated with 4G Using Tree Routing for IoT Applications", International Journal of Innovative Research in Computer and Communication Engineering, vol. 3, iss. 1, (2015).

[11] X. Li, P. Meiping, C. Cai and H. Yi, "OPNET-based modeling and simulation of mobile Zigbee sensor networks", Peer-to-Peer Netw. Appl. Springer,

[12] S. Vancin and E. Erdem, "Design and Simulation of Wireless Sensor Network Topologies Using the ZigBee Standard", International Journal of Computer Networks and Applications (IJCNA), vol. 2, iss. 3, (2015).

[13] G. Ahmed and A. Samreen, "Cluster Chain Based Relay Nodes Assignment", International Journal Of Computer Trends And Technology (Ijctt), vol. 7, (2014).

[14] W. Akyildiz, Y. Sankarasubramaniam and E. Cayirci, "Wireless Sensor Networks: A Survey Computer Networks", Elsevier, vol. 38, (2002), pp. 393-422.

[15] T. Sharma, H. Singh and A. Sharma, "A Comparative Review on Routing Protocols in Wireless Sensor Networks", vol. 123, (2015).

[16] W. Chunming and Z. Xuemei, "Routing Algorithm Simulation of the Patient Monitoring System Based on ZigBee", International Conference on Networking and Digital Society, proceeding of IEEE, (2010), pp. 26-29.

[17] S. Nourildean, "A Study of ZigBee Network Topologies for Wireless Sensor Network with One Coordinator and Multiple Coordinators", Tikrit Journal of Engineering Sciences, vol. 19, pp. 65-81, (2012).

[18] M. Kasraoui, A. Cabani and J. Mouzna, "Improvement of Zigbee Routing Protocol" 2012 IEEE International Conference on Green Computing and Communications, Conference on Internet of Things, and Conference on Cyber, Physical and Social Computing, proceeding of IEEE, (2012), pp. 788-793.

[19] R. Rajagopalan and A. Dahlstrom, "Performance Analysis of Routing Protocols in Zigbee Non- Beacon Enabled WSNs", the internet of things: RFID, WSN and beyond, proceeding of IEEE, (2013).

[20] J. Singh and R. Mahajan, "Performance Analysis of AODV and OLSR Using OPNET", International Journal of Computer Trends and Technology (IJCTT), vol. 5, no. 3, (2013).

[21] S. Kaur, S. Singh and G. Singh, "Examine the Performance of different Topologies using Opnet 14.5 in ZigBee Sensor Network", International Journal of Computer Applications (0975 - 8887), vol. 108, no. 7, (2014).

[22] A. Malek, C. Li and L. Li, "Improving ZigBee AODV Mesh Routing Algorithm Topology and Simulation Analysis", TELKOMNIKA Indonesian Journal of Electrical Engineering, vol. 12, no. 2, (2014), pp. 1528- 1535.

[23] A. Hussein and G. Samara, "Coordinator Location Effects in AODV Routing Protocol in ZigBee Mesh Network", International Journal of Computer Applications (0975 - 8887), vol. 127, no. 8, (2015).

[24] K. Kalaivani. "Zigbee Sensor Network Integrated with 4G Using Tree Routing for IoT Applications", International Journal of Innovative Research in Computer and Communication Engineering, vol. 3, iss. 1, (2015).

[25] X. Li, P. Meiping, C. Cai and H. Yi, "OPNET-based modeling and simulation of mobile Zigbee sensor networks", Peer-to-Peer Netw. Appl. Springer. 
[26] S. Vancin and E. Erdem, "Design and Simulation of Wireless Sensor Network Topologies Using the ZigBee Standard", International Journal of Computer Networks and Applications (IJCNA), vol. 2, iss. 3, (2015).

[27] G. Ahmed and A. Samreen, "Cluster Chain Based Relay Nodes Assignment" International Journal of Computer Trends and Technology (Ijctt), vol. 7, (2014).

[28] W. Akyildiz, Y. Sankarasubramaniam and E. Cayirci, "Wireless Sensor Networks: A Survey Computer Networks", Elsevier, vol. 38, (2002), pp. 393-422.

[29] T. Sharma, H. Singh and A. Sharma, "A Comparative Review on Routing Protocols in Wireless Sensor Networks", vol. 123, (2015).

[30] N. Benaouda and M. Mostefai, "A New Two-Level Clustering Scheme for Partitioning in Distributed Wireless Sensor Networks", International Journal of Distributed Sensor Networks, vol. 2015, (2015).

[31] N. Benaouda and M. Mostefai, "A New Two-Level Clustering Scheme For Partitioning In Distributed Wireless Sensor Networks", International Journal Of Distributed Sensor Networks, vol. 2015, (2015) 
International Journal of Future Generation Communication and Networking Vol. 9, No. 12 (2016) 